\title{
Wave-packet approach to transport properties of carrier coupled with intermolecular and intramolecular vibrations of organic semiconductors
}

\author{
Hiroyuki Ishii, ${ }^{1, *}$ Keisuke Honma, ${ }^{1}$ Nobuhiko Kobayashi, ${ }^{1}$ and Kenji Hirose ${ }^{2}$ \\ ${ }^{1}$ Institute of Applied Physics and Tsukuba Research Center for Interdisciplinary Materials Science, University of Tsukuba, \\ 1-1-1 Tennodai, Tsukuba, Ibaraki 305-8573, Japan \\ ${ }^{2}$ Green Innovation Research Laboratories, NEC Corporation, 34 Miyukigaoka, Tsukuba, Ibaraki 305-8501, Japan
}

(Received 30 December 2011; published 13 June 2012)

\begin{abstract}
We present a methodology to study the charge-transport properties of organic semiconductors by the timedependent wave-packet diffusion method, taking the polaron effects into account. As an example, we investigate the transport properties of single-crystal pentacene organic semiconductors coupled with inter- and intramolecular vibrations within the mixed Holstein and Peierls model, which describes both hopping and bandlike transport behaviors due to small and large polaron formations. Taking into account static disorders, which inevitably exist in the molecular crystals, we present the temperature dependence of charge-transport properties in competition among the thermal fluctuation of molecular motions, the polaron formation, and the static disorders.
\end{abstract}

DOI: 10.1103/PhysRevB.85.245206

PACS number(s): 72.80.Le, 73.50.-h, 71.38.Ht

\section{INTRODUCTION}

Organic semiconductors are expected to be one of the candidates for future electronic-device applications requiring structural flexibility and large area coverage with printing process. For example, representative applications include field-effect transistors (FETs), ${ }^{1-6}$ light-emitting diodes (LEDs), ${ }^{7,8}$ and solar cells. ${ }^{9-11}$ Recent technological progress in organic electronics requires us to obtain a much better fundamental understanding of the nature of their charge-transport mechanisms.

Different from inorganic materials such as silicon semiconductors, organic materials are formed with very weak van der Waals interactions between molecules. For typical organic semiconductors, the intermolecular bonding energies, called transfer integrals, are small, in the range of $10-10^{2} \mathrm{meV}, 12,13$ which is comparable to the depth of carrier-trap potentials by the static structural disorders, impurities, and grain boundaries. ${ }^{14}$ Then the charge carriers are strongly scattered and are localized in a molecule, creating a quasiparticle state, called a polaron, as a result of the strong interaction with intramolecular vibrations of the local lattice environment. The polaronic state should affect the transport properties of organic materials because the binding energy of a polaron (reorganization energy) has similar energetic orders of transfer integrals ${ }^{13}$ and thermal excitation energy $k_{B} T \simeq 25 \mathrm{meV}$ at room temperature. In fact, the incoherent molecular-to-molecular hopping of a polaron, which is a slow-moving electron coupled with lattice distortion, is observed in experiments as a thermally activated behavior of temperature-dependent mobility, where the carrier mobility is generally below $0.1 \mathrm{~cm}^{2} / \mathrm{V} \mathrm{s}$ at room temperature. ${ }^{2}$ In such a low mobility regime, the chargetransport properties have been investigated theoretically using the Marcus equation, ${ }^{15}$ taking polaron effects by the Holstein model, ${ }^{16}$ which describes the strong coupling between the intramolecular vibration and the electron.

Recent rapid progress in technology enables us to fabricate the single-crystal organic thin films and to construct the flexible thin-film transistor (TFT) devices with high carrier mobility up to $\sim 40 \mathrm{~cm}^{2} / \mathrm{V} \mathrm{s},{ }^{17}$ which presently exceeds the mobility of amorphous silicon. ${ }^{2}$ This shows that structural disorders are almost excluded from the organic TFTs. The temperature dependence of mobility is remarkably different from that of the disordered materials. In single crystals, the mobility decreases with increasing temperature, according to $\mu \propto T^{-n}$. Such power-law dependence is a typical character of coherent band transport due to delocalized carriers and originates from the scattering processes by the coupling between carriers and the intermolecular vibrations, i.e., the lattice phonons. Moreover, recent experimental measurements of Hall effects on the organic TFTs ${ }^{6,18,19}$ provide us with evidence of possible coherent charge transport in the single-crystal organic materials. On the other hand, a difficult problem arises in the coherent band picture. That is, the estimated mean free path is comparable to or shorter than the distance between adjacent molecules, which implies a breakdown of the coherent band transport.

Recent theoretical studies tackle these transport problems on the single-crystal organic materials with high mobility, based on the quantum-mechanical theory from an atomistic viewpoint. Troisi and Orlandi soloved the time-dependent Schrödinger equation of electrons coupled with the classical equation of molecular motion taking the polaron effects by the Peierls model, which describes the strong coupling between the intermolecular vibration and the electron. ${ }^{20}$ They showed that large thermal fluctuations of molecular motion are sufficient to destroy the translational symmetry of the molecular lattice, and the carrier state becomes sometimes delocalized bandlike and sometimes strongly localized. Fratini and Ciuchi also employed the Peierls model and found the simultaneous presence of band carriers and incoherent states. ${ }^{21}$ Ciuchi, Fratini, and Mayou analyzed the optical conductivity observed in experiments using the Kubo formula with the Peierls model and found an incipient electron localization caused by large dynamical lattice disorders. ${ }^{22}$ Böhlin, Linares, and Stafstöm investigated the polaron dynamics in the molecular lattice applied by a finite magnitude of electric field within the Peierls model and found the change of the transport process from an adiabatic polaron drift process to a combination of sequences of adiabatic drift and nonadiabatic hopping events. ${ }^{23}$

Although these approaches focus on the intermolecular coupling in the Peierls (large polaron) model, the intramolecular coupling described by the Holstein (small polaron) model cannot be ignored to account for the transport properties of 
single-crystal organic materials because the Holstein-type polaron has a large binding energy and describes the charge transport in the localized hopping regime due to static disorders, which inevitably exist in the molecular crystals. ${ }^{14}$ Hannewald and Ortmann have performed an analytical evaluation of mobility using the method of canonical transformation of the mixed Holstein-Peierls model. ${ }^{24,25}$ They studied the contribution of both the coherent and the incoherent scattering events on the transport properties. However, they assumed that the transport is bandlike, i.e., that the phase coherence is maintained and the scattering with phonon is infrequent. In general, this assumption is valid at much lower than room temperature.

To investigate the charge-transport properties of singlecrystal organic semiconductors at around room temperature, we develop a methodology based on the time-dependent wave-packet diffusion (TD-WPD) method $^{26}$ to apply for the mixed Holstein-Peierls model and to study the temperature dependence of charge-transport properties. In the present approach, we evaluate the carrier mobilities, mean free paths, and diffusion coefficients including both the Holstein-type and Peierls-type polaronic states on the same footing, with the use of the Kubo formula based on the quantum-mechanical calculations of electron wave-packet dynamics combined with the classical molecular-dynamics simulation. We show the temperature dependence of transport properties of organic semiconductors in competition among the thermal fluctuation of molecular motion, the polaron formation, and the static disorders.

\section{METHODOLOGY}

\section{A. TD-WPD methodology for organic materials}

To clarify the charge-transport properties from an atomistic viewpoint, we adopt a semiclassical approach for phonons assuming that the nuclear motions of the lattice are treated classically, whereas the charge-carrier dynamics is evaluated purely based on the quantum-mechanical approach. We note that this assumption has been frequently taken in studies of conductive polymers. ${ }^{27}$ The electron motion in materials is described directly by coupling the quantum-mechanical timeevolution calculation of an electron wave packet with the classical molecular lattice dynamics simulations, which is called the TD-WPD approach. ${ }^{26}$ In previous studies, we applied the TD-WPD method to inorganic materials, such as carbon nanotubes and graphene nanoribbons, which are formed by covalent bonding. We clarified the electron-phonon coupling effects on the transition of transport properties from the ballistic to diffusive transport regimes and from the coherent to incoherent transport regimes, from an atomistic viewpoint. ${ }^{26,28}$

The molecules in organic materials, on the other hand, are coupled to each other by weak van der Waals interactions, which make these materials much more flexible than inorganic materials. The carrier motions in the organic materials are affected strongly by the lattice distortions which form the so-called polaron state, a quasiparticle state coupled with electron and molecular vibrations. Therefore the charge-carrier transport must be described using different models from those for covalently bonded semiconductors. In the present study, we introduce the polaron effect into the TD-WPD methodology, and investigate the mechanism of charge-carrier transport with lattice distortion of flexible organic semiconductors. It should be noted that evaluation of the carrier mobility of organic materials is important from the viewpoint of not only basic science but also for a number of industrial applications.

We extract the time-dependent form of electron conductivity $\sigma$ of materials with volume $\Omega$ from the Kubo formula, ${ }^{26,29}$

$$
\begin{aligned}
\sigma= & \lim _{t \rightarrow+\infty} \int_{-\infty}^{+\infty} d E\left(-\frac{d f\left(E-E_{F}\right)}{d E}\right) \\
& \times e^{2} \operatorname{Tr}\left[\frac{\delta\left(E-\hat{H}_{e}\right)}{\Omega} \frac{\{\hat{x}(t)-\hat{x}(0)\}^{2}}{t}\right],
\end{aligned}
$$

where the Fermi-distribution function is represented by $f\left(E-E_{F}\right)$, the position operator of charge is written by $\hat{x}(t) \equiv \hat{U}^{\dagger}(t) \hat{x} \hat{U}(t)$ in the Heisenberg picture, and $\hat{U}(t=$ $\left.N_{t} \Delta t\right) \equiv \Pi_{n=0}^{N_{t}-1} \exp \left\{i \hat{H}_{e}(n \Delta t) \Delta t / \hbar\right\}$ is the time-evolution operator. The dynamical change of electronic states by lattice distortion due to polaron formation is included in the time-dependent Hamiltonian $\hat{H}_{e}(t)$. To reduce the calculation cost, the conductivity is computed numerically by Chebyshev polynomial development of $\hat{U}(t)$ using Haydock's recursion method. We evaluate the quantity of $\operatorname{Tr}[\cdots]$ by $\left(N / N_{w}\right) \times \sum_{n=1}^{N_{w}}\left\langle\Psi_{n}|\cdots| \Psi_{n}\right\rangle$, where $N$ and $N_{w}$ represent the total site number and the number of initial conditions, respectively. The use of wave packets, instead of using the direct eigenvectors of the Hamiltonian, allows us to perform the order- $N$ computation of transport properties for large-scale systems, ${ }^{26}$ suitable for the use of parallel computing.

The mobility of a carrier with an elementary charge $q$ is defined as the conductivity of Eq. (1) divided by the charge density, $\mu \equiv \sigma / q n$. The charge density is defined as $q n=$ $q \int d E f\left(E-E_{F}\right) v(E)$, where $v(E)$ is the density of states (DOS) given by $\operatorname{Tr}\left[\delta\left(E-\hat{H}_{e}\right) / \Omega\right]$. The diffusion coefficient $D$ is evaluated as the long-time limit of the time-dependent diffusion coefficient,

$$
\begin{aligned}
D & =\lim _{t \rightarrow+\infty} D(t) \\
& =\lim _{t \rightarrow+\infty} \frac{1}{t} \frac{\int d E f\left(E-E_{F}\right) \operatorname{Tr}\left[\delta\left(E-\hat{H}_{e}\right)\{\hat{x}(t)-\hat{x}(0)\}^{2}\right]}{\int d E f\left(E-E_{F}\right) \operatorname{Tr}\left[\delta\left(E-\hat{H}_{e}\right)\right]} .
\end{aligned}
$$

When we approximate the Fermi-distribution function as $f\left(E-E_{F}\right) \simeq \exp \left\{-\left(E-E_{F}\right) / k_{B} T\right\}$ since we consider the low carrier states of the semiconductor in the present study, we can extract the well-known Einstein relation, $\mu=q D / k_{B} T .^{30}$ The mean free path is also obtained from the diffusion coefficient, $l_{\mathrm{mfp}}=D / v$, where $v$ is the carrier velocity defined by $\lim _{t \rightarrow 0} \sqrt{D(t) / t}$.

Using the real-time molecular dynamics simulations, we describe the dynamical lattice distortions by both thermal fluctuation and reorganization upon ionization by charge carriers. When we employ the generalized coordinate system $\{\boldsymbol{Q}\}$ to describe the lattice distortion, the equation of motion for the $n$th site with mass $M$ is derived from the canonical equation $M \times d^{2} \boldsymbol{Q}_{n} / d t^{2}=-\partial E_{\text {tot }} / \partial \boldsymbol{Q}_{n}$, where the total energy $E_{\text {tot }}$ is defined by the summation of the electron energy and the molecular vibration energy. Here, for instance, $\boldsymbol{Q}_{n}$ represents 
the displacement of the $n$th molecular position $\Delta \boldsymbol{R}_{n}$ and the intramolecular distortion $\Delta \boldsymbol{u}_{n}$ in the present paper. In general, the total energy is divided into four terms: $E_{\text {tot }}=$ $E_{e}(\{\boldsymbol{\rho}\})+E_{l}^{\mathrm{pot}}(\{\boldsymbol{Q}\})+E_{l}^{\mathrm{kin}}(\{\dot{\boldsymbol{Q}}\})+E_{\mathrm{el}}(\{\boldsymbol{\rho} \cdot \boldsymbol{Q}\})$, where $E_{e}$, $E_{l}^{\mathrm{pot}}, E_{l}^{\mathrm{kin}}$, and $E_{\mathrm{el}}$ represent the electron energy, the elastic potential energy, the kinetic energy of the lattice vibration, and the electron-phonon coupling energy, respectively. Here, the electron density matrix is written as $\{\boldsymbol{\rho}\}$. We obtain the following equation of motion for the lattice vibration and distortion:

$$
M \frac{d^{2} \boldsymbol{Q}_{n}}{d t^{2}}=-\frac{\partial E_{l}^{\mathrm{pot}}(\{\boldsymbol{Q}\})}{\partial \boldsymbol{Q}_{n}}-\frac{\partial E_{\mathrm{el}}(\{\boldsymbol{\rho} \cdot \boldsymbol{Q}\})}{\partial \boldsymbol{Q}_{n}},
$$

where the first term in the right-hand side corresponds to the elastic force and the second term induces the lattice distortion due to polaron formation. The temperature $T$ is fixed by normalizing the kinetic energy of lattice vibration at each time step with the condition of $\sum_{n=1}^{N} M \dot{Q}_{n}^{2} / 2=d N k_{B} T / 2$ in the $d$-dimensional systems. Solutions of the coupled equations (1) and (3) allow us to describe the electron motion strongly coupled with both the thermal fluctuating lattice and the distortion by polaron formation. The calculation flow within the TD-WPD approach is shown later.

\section{B. Application to the Holstein-Peierls model}

To investigate the charge-carrier transport of organic semiconductors, we employ the mixed Holstein-Peierls model for the carriers interacting tightly with molecular distortion. The Holstein model ${ }^{16}$ is based on the local electron-phonon coupling which acts purely intramolecularly, i.e., at the single molecule ionized by the charge of carrier. On the other hand, intermolecular vibration influences the time dependence of transfer integrals between adjacent molecules. The resulting nonlocal coupling leads to a Peierls model such as the SuSchrieffer-Heeger model. ${ }^{27}$ We show a schematic picture of the hole transport in a single-crystal organic semiconductor in Fig. 1(a). The Hamiltonian for holes in the highest occupied molecular orbital (HOMO) band is written as

$$
\begin{aligned}
\hat{H}_{e}(t)= & \sum_{n, m} \tilde{\gamma}_{n m}^{\mathrm{HOMO}}\left[\Delta R_{n m}(t)\right]\left(\hat{c}_{n}^{\dagger} \hat{c}_{m}+\hat{c}_{m}^{\dagger} \hat{c}_{n}\right) \\
& +\sum_{n}\left\{\tilde{\varepsilon}_{n}^{\mathrm{HOMO}}\left[\Delta u_{n}(t)\right]+W_{n}\right\} \hat{c}_{n}^{\dagger} \hat{c}_{n},
\end{aligned}
$$

where the transfer integral $\tilde{\gamma}_{n m}^{\mathrm{HOMO}}$ and the orbital energy $\tilde{\varepsilon}_{n}^{\text {HOMO }}$ coupled with inter- and intramolecular lattice distortions are defined by

$$
\begin{gathered}
\tilde{\gamma}_{n m}^{\mathrm{HOMO}}\left(\Delta R_{n m}\right)=\gamma_{n m}^{\mathrm{HOMO}}+\alpha_{\mathrm{P}}^{\mathrm{HOMO}} \Delta R_{n m}, \\
\tilde{\varepsilon}_{n}^{\mathrm{HOMO}}\left(\Delta u_{n}\right)=\varepsilon_{n}^{\mathrm{HOMO}}+\alpha_{\mathrm{H}}^{\mathrm{HOMO}} \Delta u_{n} .
\end{gathered}
$$

Here, $\gamma_{n m}^{\text {HOMO }}$ is the bare transfer integral between the $n$th and $m$ th molecules, and $\alpha_{\mathrm{P}}^{\mathrm{HOMO}}$ is the Peierls-type electron-phonon coupling constant. When we represent the displacement of the $n$th molecule as $\Delta \boldsymbol{R}_{n}$, the change of intermolecular distance is given by $\Delta R_{n m}=\left|\left(\boldsymbol{R}_{m}^{e}+\Delta \boldsymbol{R}_{m}\right)-\left(\boldsymbol{R}_{n}^{e}+\Delta \boldsymbol{R}_{n}\right)\right|-\mid \boldsymbol{R}_{m}^{e}-$ $\boldsymbol{R}_{n}^{e} \mid$. Here $\boldsymbol{R}_{n}^{e}$ is the equilibrium position of the molecule. The operators $\hat{c}_{n}$ and $\hat{c}_{n}^{\dagger}$ are the annihilation and creation operators of a hole at the HOMO with the orbital energy $\varepsilon_{n}^{\mathrm{HOMO}}$. The Holstein-type electron-phonon coupling constant (a)
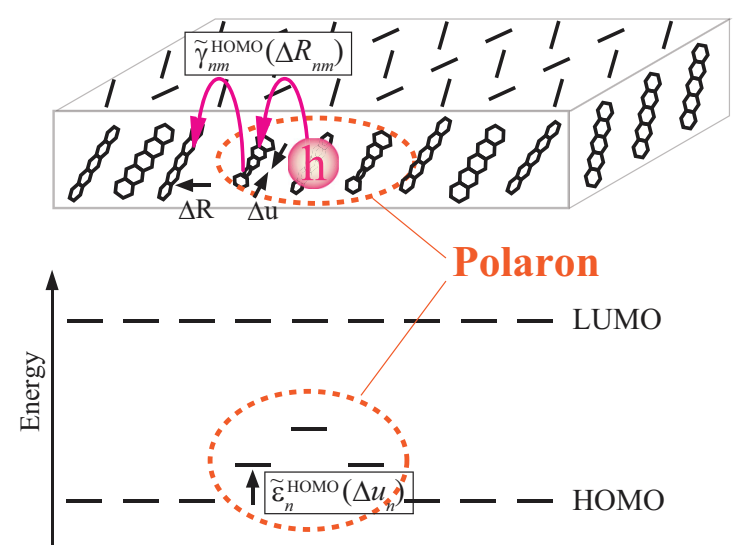

(b)

$$
\begin{aligned}
& \text { (0). Input of initial conditions at } t=0 \\
& |\Psi(0)\rangle \quad\{\Delta R(0)\} \quad\{\Delta u(0)\}
\end{aligned}
$$

(2). Wave-packet dynamics from $t$ to $t+\Delta t$

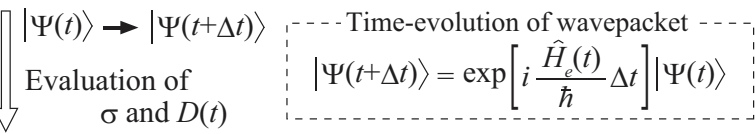

(3). Molecular dynamics from $t$ to $t+\Delta t$

$$
\begin{array}{l:l}
\Delta \mathrm{R}(t) \rightarrow \Delta \mathrm{R}(t+\Delta t) & \mathrm{d} \mathrm{d}^{2} \Delta R \\
\mathrm{~d} t^{2} & =-\frac{\mathrm{d} E_{t o t}(\{\rho\},\{\Delta R\},\{\Delta u\})}{\mathrm{d} \Delta R}
\end{array}
$$

FIG. 1. (Color online) (a) Schematic picture of the hole transport in organic semiconductors with Holstein-type and Peierls-type electron-phonon couplings. (Upper panel) Transfer energy between adjacent molecules modulated from $\gamma^{\mathrm{HOMO}}$ to $\tilde{\gamma}^{\mathrm{HOMO}} \equiv\left(\gamma^{\mathrm{HOMO}}+\right.$ $\alpha_{\mathrm{P}}^{\mathrm{HOMO}} \Delta R$ ) by the bond-length change $\Delta R$ due to both thermal fluctuation and polaron formation induced by Peierls-type coupling. (Lower panel) The HOMO and LUMO levels of each molecule in the organic semiconductor. The intramolecular deformation by reorganization, which is represented by $\Delta u$ in the upper panel, changes the HOMO level by Holstein-type coupling. (b) Flowchart of numerical computation according to the TD-WPD methodology in the case of the Holstein-Peierls model.

is represented by $\alpha_{\mathrm{H}}^{\mathrm{HOMO}}$. In the present paper, we replace the intramolecular displacement $\Delta \boldsymbol{u}_{n}$ with the effective scalar coordinate $\Delta u_{n} \cdot{ }^{38}$ The information of the atomistic configuration is included into the effective one. As a result, we can express the shift of orbital energy by $\left\{\alpha_{\mathrm{H}}^{\mathrm{HOMO}} \Delta u_{n}\right\}$. In addition, we also consider the effect of static disorder, which inevitably exists in the molecular crystals. We introduce the Anderson-type disorder potentials $W_{n}$, which modulate the on-site orbital energies randomly within the energy width $[-W / 2,+W / 2] .^{28}$

As for the molecular dynamics, we treat the dynamics of molecular lattice within the classical theory. When the elastic energies for both the inter- and intramolecular deformations are approximated by a harmonic form, we obtain the 
Hamiltonian of molecular lattice as follows:

$$
\begin{aligned}
\hat{H}_{l}(t)= & \sum_{n} \frac{1}{2} M\left\{\frac{d \Delta \boldsymbol{R}_{n}(t)}{d t}\right\}^{2}+\sum_{n, m} \frac{1}{2} K_{\mathrm{P}}\left\{\Delta R_{n m}(t)\right\}^{2} \\
& +\sum_{n} \frac{1}{2} K_{\mathrm{H}}\left\{\Delta u_{n}(t)\right\}^{2},
\end{aligned}
$$

where the inter- and intramolecular elastic constants represent $K_{\mathrm{P}}$ and $K_{\mathrm{H}}$, respectively. The total Hamiltonian is given by $\hat{H}_{\text {tot }}(t) \equiv \hat{H}_{e}(t)+\hat{H}_{l}(t)$, and we can obtain the total energy as

$$
\begin{aligned}
E_{\mathrm{tot}}= & \int d E f\left(E-E_{F}\right) \operatorname{Tr}\left[\delta\left(E-\hat{H}_{e}\right) \hat{H}_{\mathrm{tot}}\right] \\
= & \sum_{n, m} \tilde{\gamma}_{n m}^{\mathrm{HOMO}}\left(\Delta R_{n m}\right)\left\{\rho_{n m}(t)+\rho_{m n}(t)\right\} \\
& +\sum_{n} \tilde{\varepsilon}_{n}^{\mathrm{HOMO}}\left(\Delta u_{n}\right) \rho_{n n}(t)+\sum_{n} \frac{1}{2} M\left\{\frac{d \Delta \boldsymbol{R}_{n}(t)}{d t}\right\}^{2} \\
& +\sum_{n, m} \frac{1}{2} K_{\mathrm{P}}\left\{\Delta R_{n m}(t)\right\}^{2}+\sum_{n} \frac{1}{2} K_{\mathrm{H}}\left\{\Delta u_{n}(t)\right\}^{2}
\end{aligned}
$$

where $\rho_{n m} \equiv \int d E f\left(E-E_{F}\right) \operatorname{Tr}\left[\delta\left(E-\hat{H}_{e}\right) \hat{c}_{n} \hat{c}_{m}^{\dagger}\right]$ is the density matrix element. Replacing $\boldsymbol{Q}_{n}$ by $\Delta \boldsymbol{R}_{n}$ in Eq. (3), we derive the equation of molecular motion, $M \times d^{2} \Delta \boldsymbol{R}_{n} / d t^{2}=$ $-\partial E_{\text {tot }} / \partial \Delta \boldsymbol{R}_{n}$. On the other hand, as for the intramolecular distortion, we determine $\Delta u_{n}$ by the variational principle for the minimum total energy, $\partial E_{\text {tot }} / \partial \Delta u_{n}=0$, resulting in $\Delta u_{n}(t)=\left(\alpha_{\mathrm{H}} / K_{\mathrm{H}}\right)\left\{\rho_{n n}(t) / \sum_{m} \rho_{m m}(t)\right\}$.

We show the schematic flowchart for the Holstein-Peierls model in Fig. 1(b). First, we determine the Hamiltonian $\hat{H}_{e}(t)$ of electrons for the given inter- and intramolecular configurations of material at time $t$. Using the Hamiltonian, we create the time-evolution operator of an electron motion defined by $\exp \left\{-i \hat{H}_{e}(t) \Delta t / \hbar\right\}$. Then we evaluate the conductivity $\sigma$ of Eq. (1) and the time-dependent diffusion coefficient $D(t)$ of Eq. (2). Here, the Fermi energy $E_{F}$ is determined to satisfy the charge neutrality condition that the electron number in the lowest unoccupied molecular orbital (LUMO) band is equal to the hole number in the HOMO band. Then we obtain the inter- and intramolecular configurations at the next time step using the molecular lattice dynamics simulation based on the velocity Verlet algorithm, including the effect of not only the thermally excited molecular vibration but also the polaronic lattice distortions. From the renewed inter- and intramolecular configurations, we can extract the Hamiltonian at the next time step, $\hat{H}_{e}(t+\Delta t)$. Repeating these processes, we take into account the complicated trajectory of an electron motion in its induced time-dependent lattice distortion.

Here we apply the above TD-WPD method to the transport properties of single-crystal pentacene thin films. The physical quantities used here, such as electron-phonon coupling constants and elastic constants, are extracted from the calculations based on the density functional theory (DFT), including the van der Waals interactions for the energy (DFT-D approach). ${ }^{31}$ These quantities are in good agreement with some experimental observations. ${ }^{32}$ Although the various two-dimensional organic thin films can be treated with the present approach, we consider for simplicity a one-dimensional stack of 1600 pentacene molecules in the present work. This is justified since the transport properties of organic semiconductors are strongly anisotropic in real space. We obtain the bare transfer integrals between adjacent molecules $\gamma^{\mathrm{HOMO}}=-75 \mathrm{meV}$ and $\gamma^{\text {LUMO }}=-125 \mathrm{meV}$ by fitting the bandwidth of a three-dimensional pentacene crystal computed by the DFT calculations (see Ref. 33 for details) with the bandwidth $4 \gamma$ of the one-dimensional model used here. We take the HOMO and LUMO energy levels of an isolated pentacene molecule as $\varepsilon^{\mathrm{HOMO}}=-4.60 \mathrm{eV}$ and $\varepsilon^{\mathrm{LUMO}}=-2.384 \mathrm{eV}$ at the B3LYP/6-31G(d) level, ${ }^{34}$ respectively. The Peierls-type electron-phonon coupling is estimated by $\alpha_{\mathrm{P}} \equiv d \gamma / d \Delta R$ at the equilibrium molecular position, where the bond-lengthdependent $\gamma$ is calculated by the dimer-splitting approach ${ }^{12,35}$ at the DFT-D/B3LYP-D3/6-31G(d) level. For these quantities, we use $\alpha_{\mathrm{P}}^{\mathrm{HOMO}}=104 \mathrm{meV} / \AA$ and $\alpha_{\mathrm{P}}^{\mathrm{LUMO}}=112 \mathrm{meV} / \AA .^{36}$ The Peierls-type elastic constant can be estimated as $K_{\mathrm{P}}=$ $2.19 \mathrm{eV} / \AA^{2}$ by fitting the computed bond-length-dependent total energy $U(\Delta R)$ with the parabolic form $U=K_{\mathrm{P}}(\Delta R)^{2} / 2$ around the equilibrium molecular position. ${ }^{32}$ These are in good agreement with previously reported values $\left(\alpha_{\mathrm{P}}^{\mathrm{HOMO}}=\right.$ $86-174 \mathrm{meV} / \AA$ and $\left.K_{\mathrm{P}}=1.50 \mathrm{eV} / \AA^{2}\right) .{ }^{20}$ The intermolecular vibration has a continuous phonon band structure $\hbar \omega_{\mathrm{P}}(q)=$ $\hbar \sqrt{2 K_{\mathrm{P}}(1-\cos q a) / M}$ with a bandwidth of $3.8 \mathrm{meV}$, where the bond length is $a=5.16 \AA$ and the mass of a pentacene molecule is $M=4.151 \times 10^{-24} \mathrm{~kg}$. This phonon dispersion is in good agreement with an acoustic phonon branch of oligoacene crystals. ${ }^{37}$ By coupling the wave-packet dynamics with the classical molecular dynamics, we can take various phonon modes from $q=0$ to $\pm \pi / a$ thermally excited at a finite temperature to study the realistic phonon-scattering effects on the charge-transport properties. ${ }^{26}$ The Holstein-type electron-phonon coupling and the elastic constant referred to in Ref. $38, \alpha_{\mathrm{H}}^{\mathrm{HOMO}}=-93 \mathrm{meV}$ and $K_{\mathrm{H}}=92 \mathrm{meV}$, are also estimated using the DFT calculations at the B3LYP/6-31G(d) level. ${ }^{34}$ Here, we employ the dimensionless-effective-scalar coordinate $\Delta u_{n}$ as the intramolecular distortion. We confirmed that when the effective coordinate $\Delta u$ is equal to 1 , the relaxation energy defined by $K_{\mathrm{H}}(\Delta u)^{2} / 2$ and the binding energy of the Holstein-type polaron are in good agreement with those reported by other theoretical studies. ${ }^{12,38}$

As for the numerical computation of the time evolution of wave packets combined with the molecular dynamics within the TD-WPD methodology, we employ a time step $\Delta t=$ $2 \times h /(1 \mathrm{eV}) \simeq 8.272$ fs for stacked pentacene molecules. The summation up to 20th order of the Chebyshev polynomial is sufficient to ensure convergence. We evaluate Eqs. (1) and (2) using 256 initial conditions for wave packets of localized random phase states, ${ }^{39}$ distributions of static disorders, and Maxwell velocity distributions of molecules.

\section{CALCULATION RESULTS}

\section{A. Polaron formation energy}

At first, we investigate the electron-phonon coupling effect on polaron formation of single-crystal organic semiconductors without both thermal fluctuations and static disorders. The polaron state is achieved by self-consistent calculations to minimize the total energy $E_{\text {tot }}$ with respect to the intra- and the intermolecular displacements, i.e., $\Delta u_{n}$ and $\Delta R_{n}$, under 

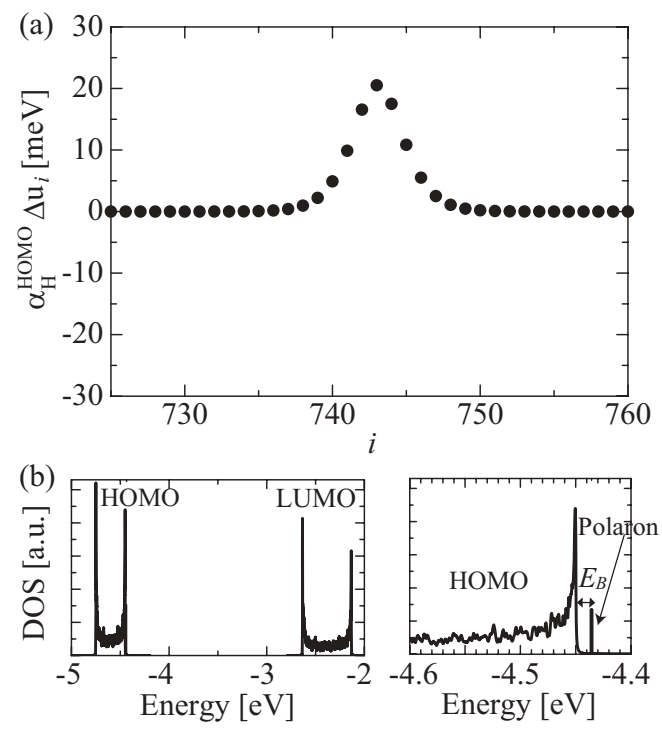

FIG. 2. (a) Change of the HOMO levels due to the Holstein-type coupling as a function of molecular number $i$. The electron-formed polaron state is localized around $i=743$ rd molecule. (b) (Left) DOS for the whole HOMO and LUMO bands. (Right) DOS of the HOMO states in energy widths from -4.6 to $-4.4 \mathrm{eV}$. A discrete energy level of polaron state is created above the HOMO band as indicated by the arrow. The binding energy $E_{B}$ is defined as the energy difference between the polaron level and the top of the HOMO band.

the condition of which the length of the molecular lattice was kept fixed. ${ }^{23,27}$ In the present calculation, we consider a carrier with spin degeneracy in a HOMO band composed of the 1600 pentacene molecular lattice, resulting in the formation of a bipolaron state. We confirm that this system size is enough to converge the calculated binding energy and the eigenstate of polarons.

\section{Holstein-type coupling effects}

First, we focus on the Holstein-type electron-phonon coupling, which describes the interaction between the electron and intramolecular distortion inducing the energy shift of the HOMO levels. Figure 2(a) shows that the change of HOMO level of the $i$ th molecule attains $\sim 20 \mathrm{meV}$ at its maximum. The obtained spatial distribution of the polaron state is localized in the range of $50 \AA$ ( 10 molecules) around $i=743$ rd molecule. We show the energy spectrum of the DOS of the whole HOMO and LUMO bands in Fig. 2(b). The spectrum shows the van Hove singularities since we employ the one-dimensional model. In the right panel of Fig. 2(b), we can see a discrete polaron state in the energy spectrum of the DOS located above the HOMO band. The binding energy $E_{B}$ is evaluated as $14.3 \mathrm{meV}$, which is the same energetic order of transfer integrals $\gamma$ and the thermal excitation energy $k_{B} T$ at around room temperature. This implies that the Holstein-type coupling affects the carrier transport properties through the polaron formation.

In Table I, we present the calculated polaron binding energies for three different models. One is the Holstein-Peierls model discussed above, and the others are the Peierls model where $\alpha_{\mathrm{P}} \neq 0$ and $\alpha_{\mathrm{H}}=0$, and the Holstein model where $\alpha_{\mathrm{P}}=0$ and $\alpha_{\mathrm{H}} \neq 0$. We observe significant differences among
TABLE I. Polaron binding energies by the Peierls, Holstein, and Holstein-Peierls models.

\begin{tabular}{lccc}
\hline \hline & Peierls & Holstein & Peierls-Holstein \\
\hline Binding energy $E_{B}(\mathrm{meV})$ & 1.3 & 7.5 & 14.3 \\
\hline \hline
\end{tabular}

these models. We see that the Holstein-Peierls model gives rise to a much larger binding energy than the Holstein or the Peierls model. These calculated results indicate that we have to take into account both the Holstein-type and Peierls-type electron-phonon couplings simultaneously when we study the transport properties of organic semiconductors.

\section{Peierls-type coupling effects and thermal fluctuation}

Next, we investigate the Peierls-type electron-phonon coupling effects on the transfer integrals. The Peierls-type coupling describes the interaction between the electron transfer integral and the intermolecular vibration, called the lattice phonon. Figure 3(a) shows the Peierls-type coupling effects on the transfer integrals of HOMO orbitals between adjacent molecules $\tilde{\gamma}_{i, i+1}^{\mathrm{HOMO}}$ as a function of molecular number $i$. Here, we consider the low-temperature limit $(T \rightarrow 0)$ to exclude the thermal fluctuation effects in the molecular dynamics
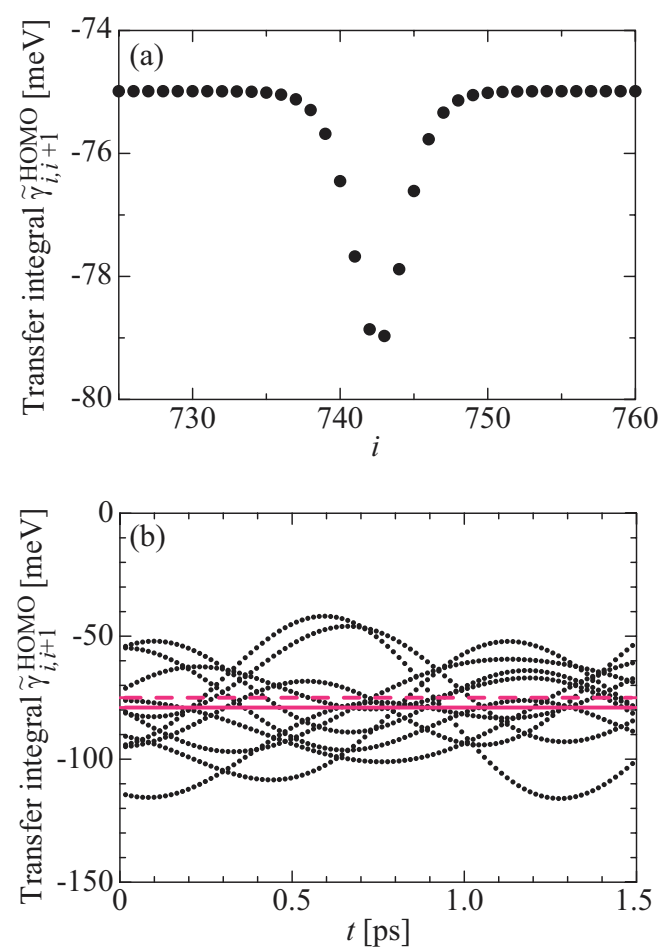

FIG. 3. (Color online) (a) The Peierls-type electron-phonon coupling effects on transfer integrals between two HOMO orbitals $\tilde{\gamma}_{i, i+1}^{\mathrm{HOMO}}$ as a function of molecular number $i$. In the calculation, the Holstein-type coupling is also included simultaneously. The electron accompanying the polaron state is localized around the $i=743 \mathrm{rd}$ molecule. (b) Time-dependent transfer integrals induced by the thermal fluctuation at $T=300 \mathrm{~K}$. For comparison, the transfer integral without the electron-phonon couplings $\left(\alpha_{\mathrm{H}}^{H}=\alpha_{\mathrm{P}}^{H}=0\right)$ is represented by a red dashed line, and the maximum value of transfer integrals enhanced by polaronic distortion is shown by a red straight line. 
simulations. We see that the transfer integrals are enhanced from $-75 \mathrm{meV}$ up to $-79 \mathrm{meV}$ by shrinking of the molecular bond length, resulting in the formation of a Peierls-type large polaron.

Since the organic semiconductor devices are operated at room temperature, it is essential to consider how the thermal fluctuations of molecular motions, as well as the polaron formation, affect the transfer integrals. Figure 3(b) shows the time-dependent transfer integrals for several bonds at $300 \mathrm{~K}$. For comparison, the transfer integral without any distortion, $\Delta R_{n m}=0$, is shown by the red dashed line. The thermal lattice vibrations give rise to a large dynamic disorder in the intermolecular transfer integrals, whereas polaron formation gives a subtle contribution to the change of transfer integral, shown by the red solid line. The amplitude of the thermally fluctuating transfer integrals reaches $\sim 80 \mathrm{meV}$, which is comparable to the magnitude of the long-time averaged transfer integrals $\tilde{\gamma}^{\mathrm{HOMO}}$, the thermal exciation energy $k_{B} T$, and the polaron binding energy $E_{B}$. These calculated results indicate that the charge-transport properties of organic semiconductors are determined in the subtle balance of energies among the thermal fluctuation of molecular motion, the thermal excitation, and the polaron formation.

\section{B. Inter- and intramolecular vibration effects on intrinsic charge transport}

In this section, we study the intrinsic charge-transport properties of single-crystal organic semiconductors, excluding the static disorders, i.e., $W=0$, and compute the time evolution of wave packets. This would give us a possible maximum value of the mobility. Figure 4(a) shows the calculated carrier mobility $\mu$ as a function of temperature $T$. The mobility decreases monotonically with increasing temperature approximately by the power-law dependence, which shows apparent evidence of the bandlike transport. ${ }^{40}$ Similar power-law temperature dependence of mobility has been reported in recent theoretical works. ${ }^{20,21}$ The present calculated results show that the intrinsic mobility reaches $\sim 100 \mathrm{~cm}^{2} / \mathrm{V} \mathrm{s}$ at around room temperature and increases up to $300 \mathrm{~cm}^{2} / \mathrm{V} \mathrm{s}$ by decreasing the temperature to $100 \mathrm{~K}$. It should be noted that we cannot take into account the bandwidth narrowing induced by the quasiparticle picture of polaron, ${ }^{41}$ since we treat the phonon vibrations based on the classical theory. The band narrowing effect can be discussed using the canonical transformation technique in whole quantum approach. ${ }^{24,25}$ Furthermore it is difficult for us to investigate a lattice heating problem induced by the phonon emission process. However, we consider these difficulties negligible in a low-bias voltage and in the temperature range of $50-400 \mathrm{~K}$ discussed in this paper. ${ }^{20}$

Let us see the time dependence of diffusion coefficient $D(t)$, which reflects the carrier motions through its time-dependent behavior. For example, $D(t)$ increases monotonically with time $t$ when the system is in the ballistic transport regime, whereas $D(t)$ saturates to a constant maximum value in the diffusive transport regime. In cases where the effects of static disorders become significant, the carrier is spatially localized and $D(t)$ becomes zero in the long-time limit. ${ }^{26}$ Figure 4(b) shows the calculated $D(t)$ for several temperatures from

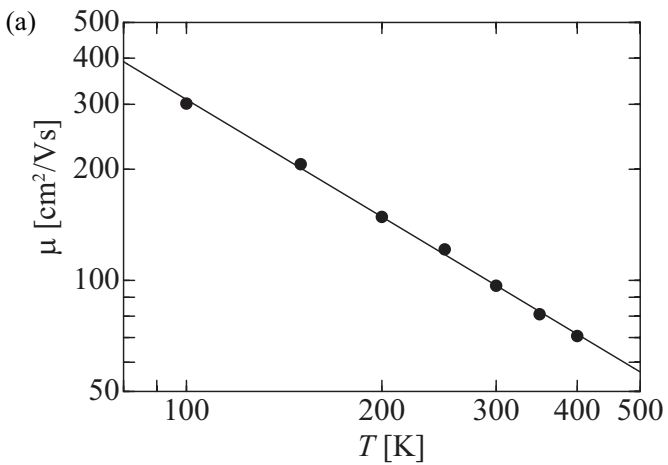

(b)

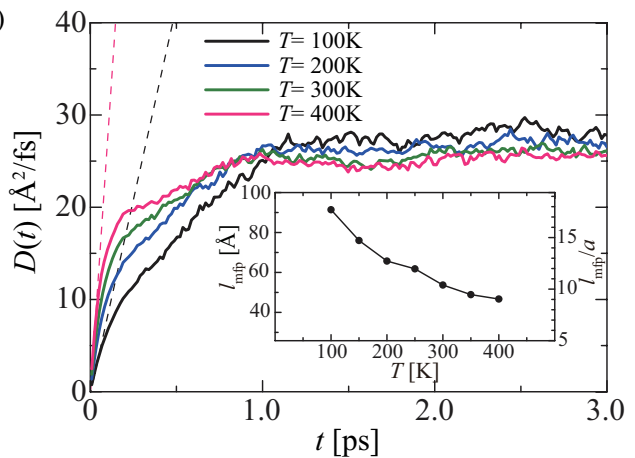

FIG. 4. (Color online) (a) The intrinsic carrier mobility $\mu$ as a function of temperature $T$ in the case of no static disorders. $\mu$ decreases monotonically with increasing $T$ and reaches $\sim 100 \mathrm{~cm}^{2} / \mathrm{V} \mathrm{s}$ at around room temperature. (b) (Main panel) Time-dependent diffusion coefficients $D(t)$ for several temperatures from 100 to $400 \mathrm{~K}$. The time-dependent behaviors show the typical diffusive transport characters without static disorders. Dashed lines represent the asymptotic lines for $T=100$ and $400 \mathrm{~K}$, respectively, whose slopes are equal to the squared carrier velocity $v^{2}$. (Inset) $l_{\mathrm{mfp}}$ as a function of $T$. The ratios of $l_{\mathrm{mfp}}$ to $a$ are also shown in the right axis.

100 to $400 \mathrm{~K}$ without static disorders. The behavior of the time dependence of $D(t)$ shows that the intrinsic carrier motion in single-crystal organic semiconductors has a typical diffusive transport character.

The mean free path $l_{\mathrm{mfp}}$, which is important to understanding the transport mechanism, can be evaluated from the diffusion coefficients as $l_{\mathrm{mfp}}=D / v$, where the carrier velocity is given by $v=\lim _{t \rightarrow 0} \sqrt{D(t) / t}$. If $l_{\mathrm{mfp}}$ is equivalent to or shorter than the lattice constant $a$ (the distance between adjacent molecules) as $l_{\mathrm{mfp}}<a$, then the carrier takes the hopping transport process, while the bandlike transport process is characterized as $l_{\mathrm{mfp}}$ much longer than $a$, such as $l_{\mathrm{mfp}} \gg a$. In Fig. 4(b), we show the time-dependent behaviors of $D(t)$ for $T=100$ and $400 \mathrm{~K}$. The dashed lines, the slope of $D(t)$, are equal to the squared carrier velocities $v^{2}$. The magnitude of velocity increases with the temperature, $v \propto \sqrt{T}$, since the carriers are excited thermally. From this information, we obtain $l_{\mathrm{mfp}}$ and its $T$ dependence is shown in the inset. The carrier scatterings are included in the changes of the time-dependent transfer integrals, which are induced by the thermal fluctuation of molecular motion through the Peierls-type electron-phonon coupling. Then the mean free path decreases monotonically as the temperature increases as $l_{\mathrm{mfp}}=v \tau \propto v /\left\langle\left(\Delta R_{n m}\right)^{2}\right\rangle \propto$ $v /\left(k_{B} T\right) .{ }^{21}$ For reference, the ratio of $l_{\mathrm{mfp}}$ to $a$ is plotted in the right axis. We see that the mean free path reaches ten times 


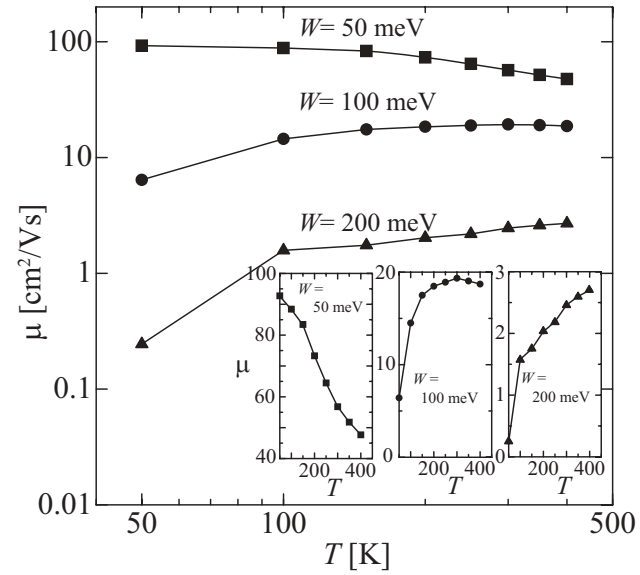

FIG. 5. Logarithmic plot of the carrier mobility $\mu$ as a function of temperature $T$ for the static disorders of $W=50,100$, and $200 \mathrm{meV}$, respectively. As $W$ increases, the $T$ dependence of $\mu$ at around room temperature changes from the nearly power-law dependence to the thermally activated behavior. (Inset) Normal linear plots of $\mu$ vs $T$ characteristics for the static disorders of $W=50$ (left), 100 (center), and $200 \mathrm{meV}$ (right).

longer than the lattice constant at around room temperature, supporting the possibility of bandlike behavior in the intrinsic transport when the static disorders are absent.

\section{Static disorder effects on charge transport}

Here, we investigate how the transport properties are affected by the static disorders $W$, which inevitably exist in the molecular crystals in terms of static defects such as crystal imperfections and the presence of impurities. Several experimental evaluations show that the potential depths of static disorders are estimated as $\sim 50 \mathrm{meV} .{ }^{14}$ Thus we change the parameter $W$ between 50 and $200 \mathrm{meV}$. We note that these static disorders are comparable to the HOMO bandwidth of the present organic semiconductors. In fact, the ratio of $W / \gamma$ HOMO ranges from 0.67 to 2.67 . Therefore, the charge-transport properties are expected to be strongly disturbed by the effects of the presence of static disorders in the various temperature regimes.

Figure 5 shows the logarithmic plot of the carrier mobility $\mu$ as a function of the temperature $T$ for several strengths of static disorders $W=50,100$, and $200 \mathrm{meV}$. In the inset, we plot the normal linear behavior of the mobility $\mu$ vs $T$. The existence of static disorders decreases the carrier mobility significantly from $100 \mathrm{~cm}^{2} / \mathrm{V} \mathrm{s}$ (no static disorder) up to $2 \mathrm{~cm}^{2} / \mathrm{V} \mathrm{s}$ ( $W=200 \mathrm{meV}$ ) around $300 \mathrm{~K}$, and more importantly their temperature dependencies are changed completely.

In the case of $W=50 \mathrm{meV}$, the magnitude of $\mu$ becomes larger than $50 \mathrm{~cm}^{2} / \mathrm{V} \mathrm{s}$ at around room temperature and $d \mu / d T$ takes negative values in the whole temperature regime studied here, showing a monotonic increase of $\mu$ as a decrease of $T$. The $T$ dependence of $\mu$ higher than $200 \mathrm{~K}$ is close to the power-law dependence seen in Fig. 4(a). On the other hand, the slope of an increase of $\mu$ becomes dull in the low $T$ regime since the scatterings by static disorders with no temperature dependence become dominant for the carrier motion. To clarify the details of the transport mechanism,
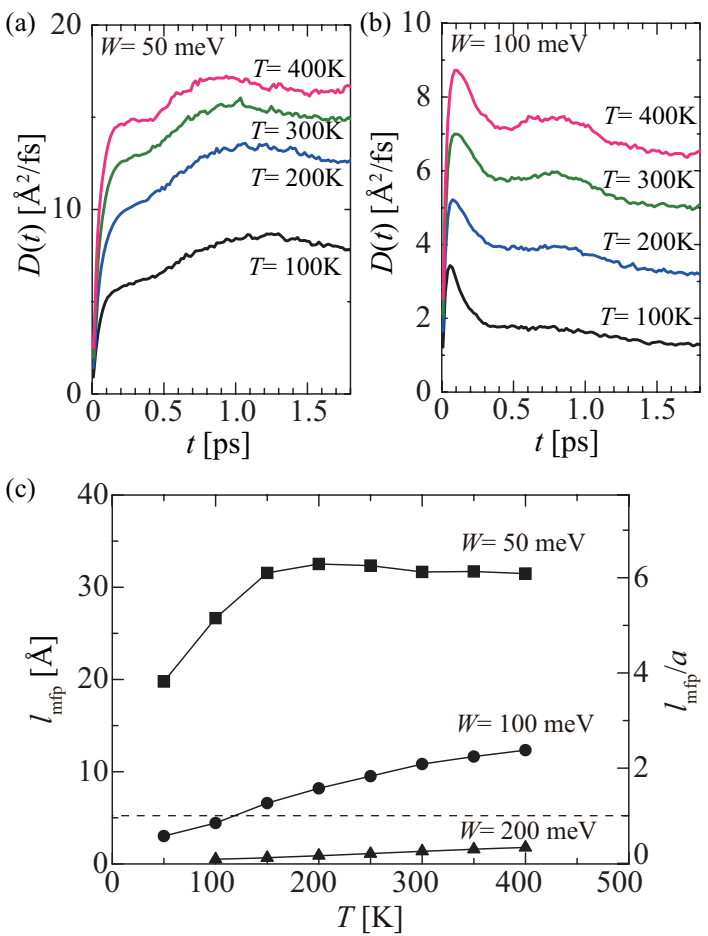

FIG. 6. (Color online) Time-dependent diffusion coefficients $D(t)$ for temperatures from 100 to $400 \mathrm{~K}$ in the presence of static disorders with (a) $W=50$ and (b) $100 \mathrm{meV}$. (c) Mean free path $l_{\text {mfp }}$ as a function of temperature $T$ for $W=50,100$, and $200 \mathrm{meV}$, respectively. The ratios of $l_{\mathrm{mfp}}$ to $a$ are also shown in the right axis.

we show the time-dependent diffusion coefficients $D(t)$ in Fig. 6(a). We see that their time dependencies are remarkably similar to those in Fig. 4(b), which indicates the realization of diffusive transport rather than hopping transport of a localized carrier. Figure $6(\mathrm{c})$, in which the mean free paths $l_{\mathrm{mfp}}$ are presented as a function of $T$, shows four to six times longer than the lattice constant $a$ in the case of $W=50 \mathrm{meV}$.

When the disorder $W$ increases to $100 \mathrm{meV}, d \mu / d T$ changes its sign $<300 \mathrm{~K}$ as shown in Fig. 5; that is to say, $\mu$ changes its behavior from bandlike in the high- $T$ regime to the thermally activated one in the low- $T$ regime. In this situation, we expect that the carrier is localized by the static disorders and induces the intramolecular distortions, resulting in a low-mobility polaron formation. From a comparison with the calculated mobility by taking only the Peierls-type coupling, we find that the presence of Holstein-type coupling, which describes the interaction between a hole and the intramolecular distortion, decreases the mobility from 11.4 to $6.4 \mathrm{~cm}^{2} / \mathrm{V} \mathrm{s}$ at $50 \mathrm{~K}$ and from 15.0 to $14.5 \mathrm{~cm}^{2} / \mathrm{Vs}$ at $100 \mathrm{~K}$. At temperatures $>150 \mathrm{~K}$, the mobility reduction by the polaron formation becomes negligible, and the mobility takes its maximum value of $19 \mathrm{~cm}^{2} / \mathrm{V} \mathrm{s}$ at $300 \mathrm{~K}$. Then $\mu$ decreases again with increasing $T$. We note that such $T$ dependence of $\mu$ has been observed in several recent experiments of organic TFTs. ${ }^{1,42}$

To clarify their transport properties, let us investigate the time dependence of $D(t)$. We see in Fig. 6(b) that $D(t)$ increases with time at first and takes the maximum value at $\sim 0.1 \mathrm{ps}$, then decreases gradually. This indicates that the charge carrier is localized by the strong static disorders, 
resulting in low mobility and thermally hopping behavior with a finite polaron formation energy. Actually, from Fig. 6(c), we can see that the $l_{\mathrm{mfp}}$ is comparable to the lattice constant $a$, which agrees well with experimental observations. ${ }^{43}$ The polaronic localization state is destroyed with increasing $T$ because the thermal excitation energy beyond the polaron binding energy is given to the carrier.

When the static disorders become more significant, up to $W=200 \mathrm{meV}$, the magnitude of $\mu$ takes a much lower value of $\sim 1 \mathrm{~cm}^{2} / \mathrm{V} \mathrm{s}$ in comparison with those for $W=50$ and $100 \mathrm{meV}$. The sign of $d \mu / d T$ becomes positive in the whole temperature regime, as shown in Fig. 5. In this situation, the carrier is trapped tightly by the disorders, therefore the calculated $l_{\mathrm{mfp}}$ in Fig. 6(c) is less than the lattice constant $a$. The evaluated mean free path of carrier is shorter than the moleculeto-molecule distance, which renders the concept of bandlike transport meaningless. The carrier transport properties are very close to typical thermally activated behaviors, which have been observed for the organic TFTs with low quality. ${ }^{2}$

Finally, we study the time dependence of carrier motion in organic semiconductors. Figure 7 shows the propagation length of carrier as a function of time, which is defined by $L(t) \equiv \sqrt{D(t) \times t}$. We also plot the ideal time-dependent behaviors of $L(t)$ by thin solid lines in the limits of the ballistic $(L \propto t)$, the diffusive $(L \propto \sqrt{t})$, and the localized (time-independent) characters, respectively. When the time exceeds $t \simeq 30 \mathrm{fs}$, the carrier begins to be scattered both by the electron-phonon couplings and by the static disorders, and its time dependence deviates gradually from the ballistic behavior. The localized nature of carriers is enhanced in the presence of static disorders. We find that the estimated localization length is $\sim 10 \AA$ in the case of $W=200 \mathrm{meV}$. Note that the crystal structure of organic molecular systems is considered as

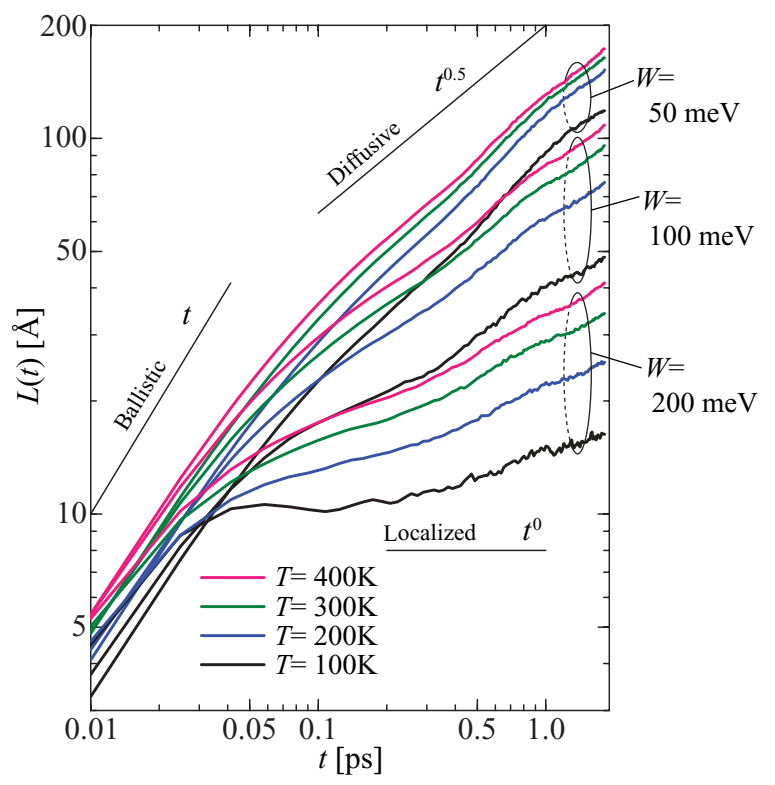

FIG. 7. (Color online) Propagation length $L(t) \equiv \sqrt{D(t) \times t}$, as a function of time for several $T$ 's and $W$ 's. We plot the ideal time-dependent behavior of $L$ in the limit of the ballistic $(L \propto t)$, the diffusive $\left(L \propto t^{0.5}\right)$, and the localization (time-independent) characters, respectively. quasi-one-dimensional due to its highly anisotropic character. For a purely one-dimensional model, the localization effect sets in more efficiently, therefore the above estimation should be an upper bound to the localization length. As $T$ increases or $W$ decreases, the carrier begins to move and its motion changes from the thermally activated hopping transport due to the localized carrier to the diffusive transport character.

\section{SUMMARY}

In summary, we have developed a methodology to calculate the carrier transport coupled with the inter- and the intramolecular vibrations of organic semiconductors based on the TD-WPD method. In this methodology, we carry out the quantum-mechanical time-evolution calculations of wave packets and the classical molecular dynamics simulations simultaneously, including the inter- and intramolecular lattice distortions and the static disorders on an equal footing without any perturbative treatment. This enables us to take into account the polaron formation effects, which are considered indispensable in the carrier transport of organic semiconductors.

Using this methodology, taking both the Holstein-type (small) and Peierls-type (large) polaron formations, we studied the carrier mobilities and the mean free paths of singlecrystal pentacene organic semiconductors, focusing on their temperature dependence in competition among the thermal fluctuation of molecular motion, the polaron formation, and the static disorders.

When the static disorders, such as crystal imperfections, and the presence of impurities are absent in the idealized situations, we found that the intrinsic mobility reaches $\sim 100 \mathrm{~cm}^{2} / \mathrm{V} \mathrm{s}$ at around room temperature and increases up to $300 \mathrm{~cm}^{2} / \mathrm{V} \mathrm{s}$ with decreasing the temperature to $100 \mathrm{~K}$, which follows the power-law dependence. We found that this is mainly due to the transfer-integral modulation originating from the thermal fluctuation of molecular motion, whereas the polaron formation gives a subtle contribution to the transport properties. As the static disorders increase, the carrier becomes localized and couples with the intramolecular distortions, forming the polaron state. Therefore, the magnitude of mobility is dramatically decreased and its temperature dependence changes gradually from the bandlike transport behavior $d \mu / d T<0$ to the thermally activated hopping transport behavior $d \mu / d T>0$. We note that such transport behaviors have been observed frequently in experiments. ${ }^{1,42}$ The present calculated results indicate that the thermal fluctuation, the polaron formation, and the static disorders provide important contributions to the understanding of the transport mechanism of realistic organic semiconductors.

\section{ACKNOWLEDGMENTS}

We would like to acknowledge J. Takeya and T. Uemura for valuable comments and suggestions. This work was supported by the Grant-in-Aid for Scientific Research from the MEXT, Japan. Numerical calculations were performed at the Center for Computational Sciences, University of Tsukuba, and the Supercomputer Center, ISSP, University of Tokyo. 
*ishii@bk.tsukuba.ac.jp

${ }^{1}$ S. F. Nelson, Y.-Y. Lin, D. J. Gundlach, and T. N. Jackson, Appl. Phys. Lett. 72, 1854 (1998).

${ }^{2}$ C. D. Dimitrakopoulos and P. R. L. Malenfant, Adv. Mater. 14, 99 (2002).

${ }^{3}$ V. Podzorov, V. M. Pudalov, and M. E. Gershenson, Appl. Phys. Lett. 82, 1739 (2003).

${ }^{4}$ M. Kiguchi, M. Nakayama, K. Fujiwara, K. Ueno, T. Shimada, and K. Saiki, Jpn. J. Appl. Phys. 42, L1408 (2003).

${ }^{5}$ O. D. Jurchescu, J. Baas, and T. T. M. Palstra, Appl. Phys. Lett. 84, 3061 (2004)

${ }^{6}$ J. Takeya, K. Tsukagoshi, Y. Aoyagi, T. Takenobu, and Y. Iwasa, Jpn. J. Appl. Phys. 44, L1393 (2005).

${ }^{7}$ J. H. Burroughes, D. D. C. Bradley, A. R. Brown, R. N. Marks, K. Mackay, R. H. Friend, P. L. Burns, and A. B. Holmes, Nature (London) 347, 539 (1990).

${ }^{8}$ Y. Ohmori, M. Uchida, K. Muro, and K. Yoshino, Jpn. J. Appl. Phys. 30, L1941 (1991).

${ }^{9}$ C. W. Tang, Appl. Phys. Lett. 48, 183 (1986).

${ }^{10}$ J. Xue, S. Uchida, B. P. Rand, and S. R. Forrest, Appl. Phys. Lett. 85, 5757 (2004).

${ }^{11}$ J. Y. Kim, K. Lee, N. E. Coates, D. Moses, T.-Q. Nguyen, M. Dante, and A. J. Heeger, Science 317, 222 (2007).

${ }^{12}$ V. Coropceanu, J. Cornil, D. A. da Silva Filho, Y. Oliver, R. Silbey, and J.-L. Brédas, Chem. Rev. 107, 926 (2007).

${ }^{13}$ J.-L. Brédas, D. Beljonne, V. Coropceanu, and J. Cornil, Chem. Rev. 104, 4971 (2004).

${ }^{14}$ W. L. Kalb and B. Batlogg, Phys. Rev. B 81, 035327 (2010).

${ }^{15}$ R. A. Marcus, Rev. Mod. Phys. 65, 599 (1993).

${ }^{16}$ T. Holstein, Ann. Phys. 8, 325 (1959); 8, 343 (1959).

${ }^{17}$ J. Takeya, M. Yamagishi, Y. Tominari, R. Hirahara, Y. Nakazawa, T. Nishikawa, T. Kawase, T. Shimoda, and S. Ogawa, Appl. Phys. Lett. 90, 102120 (2007).

${ }^{18}$ V. Podzorov, E. Menard, J. A. Rogers, and M. E. Gershenson, Phys. Rev. Lett. 95, 226601 (2005).

${ }^{19}$ M. Yamagishi, J. Soeda, T. Uemura, Y. Okada, Y. Takatsuki, T. Nishikawa, Y. Nakazawa, I. Doi, K. Takimiya, and J. Takeya, Phys. Rev. B 81, 161306(R) (2010).

${ }^{20}$ A. Troisi and G. Orlandi, Phys. Rev. Lett. 96, 086601 (2006); J. Phys. Chem. A 110, 4065 (2006).

${ }^{21}$ S. Fratini and S. Ciuchi, Phys. Rev. Lett. 103, 266601 (2009).

${ }^{22}$ S. Ciuchi, S. Fratini, and D. Mayou, Phys. Rev. B 83, 081202(R) (2011).

${ }^{23}$ J. Böhlin, M. Linares, and S. Stafström, Phys. Rev. B 83, 085209 (2011).

${ }^{24}$ K. Hannewald, V. M. Stojanović, J. M. T. Schellekens, P. A. Bobbert, G. Kresse, and J. Hafner, Phys. Rev. B 69, 075211 (2004); K. Hannewald and P. A. Bobbert, Appl. Phys. Lett. 85, 1535 (2004).

${ }^{25}$ F. Ortmann, F. Bechstedt, and K. Hannewald, Phys. Rev. B 79, 235206 (2009); F. Ortmann and S. Roche, ibid. 84, 180302(R) (2011).

${ }^{26}$ H. Ishii, N. Kobayashi, and K. Hirose, Appl. Phys. Express 1, 123002 (2008); Phys. Rev. B 82, 085435 (2010).
${ }^{27}$ W. P. Su, J. R. Schrieffer, and A. J. Heeger, Phys. Rev. Lett. 42, 1698 (1979); Phys. Rev. B 22, 2099 (1980).

${ }^{28}$ H. Ishii, S. Roche, N. Kobayashi, and K. Hirose, Phys. Rev. Lett. 104, 116801 (2010); H. Ishii, N. Kobayashi, and K. Hirose, Appl. Phys. Express 3, 095102 (2010).

${ }^{29}$ S. Roche and D. Mayou, Phys. Rev. Lett. 79, 2518 (1997); S. Roche, J. Jiang, F. Triozon, and R. Saito, ibid. 95, 076803 (2005).

${ }^{30}$ S. M. Sze, Semiconductor Devices: Physics and Technology, 2nd ed. (Wiley, Hoboken, NJ, 2001); D. K. Ferry, Semiconductor Transport (Taylor \& Francis, London, 2000).

${ }^{31}$ S. Grimme, J. Comput. Chem. 25, 1463 (2004).

${ }^{32}$ T. Uemura, T. Fukami, H. Ishii, N. Kobayashi, K. Hirose, and J. Takeya (unpublished).

${ }^{33}$ K. Hummer and C. Ambrosch-Draxl, Phys. Rev. B 72, 205205 (2005).

${ }^{34}$ We use the GAUSSIAN03 program at the B3LYP/6-31G(d) level: M. J. Frisch, G. W. Trucks, H. B. Schlegel, G. E. Scuseria, M. A. Robb, J. R. Cheeseman, J. A. Montgomery Jr., T. Vreven, T. Kudin, J. C. Burant, J. M. Millam, S. S. Iyengar, J. Tomasi, V. Barone, B. Mennucci, M. Cossi, G. Scalmani, N. Rega, G. A. Petersson, H. Nakatsuji, M. Hada, M. Ehara, K. Toyota, R. Fukuda, J. Hasegawa, M. Ishida, T. Nakajima, Y. Honda, O. Kitao, H. Nakai, M. Klene, X. Li, J. E. Knox, H. P. Hratchian, J. B. Cross, C. Adamo, J. Jaramillo, R. Gomperts, R. E. Stratmann, O. Yazyev, A. J. Austin, R. Cammi, C. Pomelli, J. W. Ochterski, P. Y. Ayala, K. Morokuma, G. A. Voth, P. Salvador, J. J. Dannenberg, V. G. Zakrzewski, S. Dapprich, A. D. Daniels, M. C. Strain, O. Farkas, D. K. Malick, A. D. Rabuck, K. Raghavachari, J. B. Foresman, J. V. Ortiz, Q. Cui, A. G. Baboul, S. Clifford, J. Cioslowski, B. B. Stefanov, G. Liu, A. Liashenko, P. Piskorz, I. Komaromi, R. L. Martin, D. J. Fox, T. Keith, M. A. Al-Laham, C. Y. Peng, A. Nanayakkara, M. Challacombe, P. M. W. Gill, B. Johnson, W. Chen, M. W. Wong, C. Gonzalez, and J. A. Pople, Gaussian03, Revision B. 03, Gaussian Inc., Pittsburgh, PA, 2003.

${ }^{35}$ J. L. Brédas, J. P. Calbert, D. A. da Silva Filho, and J. Cornil, Proc. Natl. Acad. Sci. USA 99, 5804 (2002).

${ }^{36}$ We use the GAMESS program at the DFT-D/B3LYP-D3/6-31G(d) level: M. W. Schmidt, K. K. Baldridge, J. A. Boatz, S. T. Elbert, M. S. Gordon, J. H. Jensen, S. Koseki, N. Matsunaga, K. A. Nguyen, S. J. Su, T. L. Windus, M. Dupuis, and J. A. Montgomery Jr., J. Comput. Chem. 14, 1347 (1993).

${ }^{37}$ I. Natkaniec, E. L. Bokhenkov, B. Dorner, J. Kalus, G. A. Mackenzie, G. S. Pawley, U. Schmelzer, and E. F. Sheka, J. Phys. C 13, 4265 (1980).

${ }^{38}$ J. Lei and Y. Shimoi, J. Phys. Soc. Jpn. 80, 034702 (2011).

${ }^{39}$ T. Markussen, R. Rurali, M. Brandbyge, and A.-P. Jauho, Phys. Rev. B 74, 245313 (2006).

${ }^{40}$ N. Karl, Synth. Met. 133, 649 (2003).

${ }^{41}$ N. Koch, A. Vollmer, I. Salzmann, B. Nickel, H. Weiss, and J. P. Rabe, Phys. Rev. Lett. 96, 156803 (2006).

${ }^{42}$ V. Podzorov, E. Menard, A. Borissov, V. Kiryukhin, J. A. Rogers, and M. E. Gershenson, Phys. Rev. Lett. 93, 086602 (2004).

${ }^{43}$ J. Takeya, C. Goldmann, S. Haas, K. P. Pernstich, B. Ketterer, and B. Batlogg, J. Appl. Phys. 94, 5800 (2003). 